\title{
Modelagem estatística para previsão de geada de radiação
}

\author{
Statistical modeling for radiative frost forecast \\ Alessandro da Silva e Rosandro Boligon Minuzzi \\ Universidade Federal de Santa Catarina \\ Brasil \\ alessandroagroufsc@yahoo.com.br; rbminuzzi@hotmail.com
}

\begin{abstract}
Resumo
O objetivo do estudo foi obter modelos estatísticos para a previsão de geada branca. Foram utilizados dados meteorológicos diários de temperatura mínima e temperatura média do ar das 18 he 00 h (TMG - Tempo Médio de Greenwich) no período de maio a agosto de 2000 a 2015, de estações localizadas nos municípios de Campos Novos, Lages, Chapecó e São Joaquim, em Santa Catarina. Foi utilizada a regressão linear simples e polinomial para correlacionar as variáveis independentes $(X)$, temperatura mínima, temperatura média, temperatura das 18 he $00 \mathrm{~h}$ (TGM) do dia anterior (Ti-1), com a variável dependente (Y), temperatura mínima do dia presente (Tmin i). $O$ indice de confiança foi utilizado para avaliar a confiabilidade da estimativa de temperatura mínima (Ti). A temperatura do ar a $00 \mathrm{~h}$ (TMG) foi a melhor variável preditora para a temperatura mínima (Tmin i). Os modelos estatísticos obtidos para previsão de temperatura mínima (Tmin i) apresentaram um desempenho 'muito bom'. Em eventos de geada branca $\left(\operatorname{Tmin} \leq 4^{\circ} \mathrm{C}\right)$, o acerto nas estimativas esteve entre $64,3 \%$ e $88,9 \%$.
\end{abstract}

Palavras-chave: Temperatura mínima; Radiação terrestre; Geada branca.

\begin{abstract}
The objective was to obtain statistical models for the prediction of hoarfrost. Were used daily weather data minimum temperature and average air temperature of $18 \mathrm{~h}$ and $00 \mathrm{~h}$ (GMT - Greenwich Mean Time) in the period May-August 2000 to 2015, of stations located in the cities of Campos Novos, Lages, Chapecó and São Joaquim, Santa Catarina. Was used Simple Linear Regression and Polynomial for correlating the independent variables $(X)$, minimum temperature, mean temperature, temperature of $18 \mathrm{~h}$ and $00 \mathrm{~h}$ (GMT) on the previous day (Ti-1), with the dependent variable $(Y)$, temperature minimum present day (Tmin i). The confidence index was used to assess the reliability of the estimated minimum temperature (Ti). The air temperature at $00 \mathrm{~h}$ (GMT) was the best predictor variable for the minimum temperature (Tmin i). Statistical models obtained for minimum temperature forecast (Tmin i) presented a performance of 'very good'. In hoarfrost events (Tmin $\leq 4{ }^{\circ} \mathrm{C}$ ), the accuracy of estimates was between $64,3 \%$ and $88,9 \%$.
\end{abstract}

Keywords: Minimum temperature; Terrestrial radiation; Hoarfrost. 


\section{Introdução}

No contexto meteorológico, a geada é caracterizada pela deposição de gelo sobre plantas e outras superfícies que estejam expostas ao relento. Sentelhas e Angelocci (2012) relatam que em noites de céu sem nebulosidade, sem vento, com baixa concentração de vapor d'água, sob domínio de uma massa de ar polar e aliada à perda de radiação terrestre, tem-se a inversão térmica, onde a temperatura de relva encontra-se abaixo da temperatura do ar, originando a geada de radiação.

Aguiar e Mendonça (2004) observaram que embora ocorra na região sudeste do Brasil, a maior incidência de geada de radiação se dá no sul do país, onde no inverno as temperaturas ficam abaixo de $0{ }^{\circ} \mathrm{C}$ com mais frequência. Em Santa Catarina as constantes entradas de massas de ar polar, fazem com que este evento não fique restrito somente ao inverno. Somando a isto, as condições de latitude, maritimidade e relevo resultam numa maior distribuição espacial pelo Estado. Araújo et al. (2009) apontaram a maior ocorrência de geadas em Santa Catarina no período de maio a agosto, sendo a maior parte de baixa intensidade $(40 \%$ a $60 \%)$ e apenas $20 \%$ a $35 \%$ de intensidade moderada.

No setor agrícola a geada está diretamente associada a prejuízos na lavoura. Para Sentelhas e Angelocci (2012) a geada de radiação não é tão danosa quanto à geada negra. Isto porque durante o processo de congelamento do vapor d'água na relva, há a liberação de calor latente, impedindo que a temperatura baixe ainda mais. Porém o congelamento dos tecidos vegetais leva a perda do potencial de turgescência, redução do volume celular, resultando na desidratação das células e, eventualmente na morte parcial ou total da planta (PERISSATO et al., 2013).

Perissato et al. (2013) relataram que a geada afeta as culturas em diferentes estádios fenológicos, citando como exemplo a Canola, que se atingida pela geada no início de seu desenvolvimento, prejudica o seu florescimento e o enchimento dos grãos, refletindo na baixa produtividade e, consequentemente na produção de óleo, utilizado na fabricação de biocombustível. Também constataram a redução na massa dos grãos de milho atingido pela geada.

Brixner et al. (2014) verificaram que em videiras da cultivar 'Cabernet Sauvignon' atingidas por geadas, os danos nos órgãos e tecidos foram visíveis, principalmente nas gemas em início de brotação, caracterizados pelo aspecto caramelado e depois seco. Outra constatação foi que em áreas de maior incidência de geadas, o ciclo fenológico da videira se estendeu. Aguiar e Mendonça (2004) relataram que mesmo para o trigo cuja geada é benéfica quando se encontra na fase vegetativa, pode ter uma lavoura inteira dizimada se esta ocorrer em fases posteriores, onde a planta torna-se sensível a baixas temperaturas.

A geada também influencia diretamente na pecuária, uma vez que afeta o crescimento das pastagens, diminuindo a oferta de alimentos e fazendo com que o produtor precise utilizar outros complementos nutricionais, como rações e silagens, o que aumenta significativamente os custos de produção. Por sua vez, esta alta atingirá diretamente o consumidor, devido à escassez de produtos como a carne e o leite, além do aumento da demanda.

No contexto de previsão, Ramos et al. (2011) estudaram a variação da temperatura ao decorrer do dia nas cidades de Petrolina-PE e Botucatu-SP, correlacionando-as aos horários de medição das estações meteorológicas. Concluíram que a medição entre as $22 \mathrm{~h} \mathrm{e} 8 \mathrm{~h}$ da manhã do dia seguinte, são os mais indicados para uso na modelagem devido a menor influência de outras condições climáticas locais.

Portanto além de influenciar na produção de matéria -prima, a geada acaba ocasionando prejuízos econômicos e sociais, tornando importante sua previsão com maior precisão, para auxiliar na adoção de medidas preventivas a curto, médio e longo prazo. Por isso, este estudo teve como objetivo obter modelos estatísticos para previsão de geada de radiação no estado de Santa Catarina.

\section{Material e métodos}

Foram utilizados dados diários de temperatura mínima e temperatura média do ar das 18 h e 00 h (TMG - Tempo Médio de Greenwich) no período de maio a agosto de 2000 a 2015, de estações meteorológicas pertencentes ao Instituto Nacional de Meteorologia (INMET) (Tabela 1).

Para obtenção dos modelos para previsão da ocorrência de geada, foi utilizada a regressão linear simples e a polinomial, para correlacionar as variáveis independentes $(X)$ temperatura mínima (Tmin i-1), temperatura média, temperatura das $18 \mathrm{~h}$ e $00 \mathrm{~h}$ (TGM) do dia anterior (Ti1), com a temperatura mínima do dia presente (Tmin i), como a variável dependente $(Y)$.

Assim foram feitas as seguintes correlações:

- temperatura mínima (Tmin i-1) x temperatura mínima (Tmin i);

- temperatura 18 h (TMG) (18Ti-1) x temperatura mínima (Tmin i);

Tabelas 1 - Coordenadas geográficas, altitude e município a qual pertencem às estações meteorológicas utilizadas no estudo.

\begin{tabular}{c|c|c|c}
\hline Município & Latitude & Longitude & $\begin{array}{c}\text { Altitude } \\
(\mathrm{m})\end{array}$ \\
\hline Lages & $27^{\circ} 49^{\prime} \mathrm{S}$ & $49^{\circ} 56^{\prime} \mathrm{W}$ & 937 \\
São Joaquim & $28^{\circ} 18^{\prime} \mathrm{S}$ & $50^{\circ} 20^{\prime} \mathrm{W}$ & 1415 \\
Campos & $27^{\circ} 24^{\prime} \mathrm{S}$ & $51^{\circ} 12^{\prime} \mathrm{W}$ & 952 \\
Novos & $27^{\circ} 07^{\prime} \mathrm{S}$ & $52^{\circ} 37^{\prime} \mathrm{W}$ & 679 \\
Chapecó & & & \\
\hline
\end{tabular}


- temperatura $00 \mathrm{~h}$ (TMG) (00Ti-1) x temperatura mínima (Tmin i);

O software estatístico PAST 3.0 foi utilizado para avaliar a significância estatística das correlações, utilizando o coeficiente de correlação $(r)$, o coeficiente de determinação da regressão linear $\left(\mathrm{R}^{2}\right)$, o erro padrão de estimativa (EPE), o valor-p e o valor F. Os dados dos anos 2014 e 2015 foram utilizados para testar os modelos estatísticos com a regressão linear de melhor correlação (Equação 1).

$$
\mathrm{Y}=\mathrm{a}+\mathrm{bX}+\mathrm{cXn} \ldots \pm \mathrm{EPE}
$$

Os índices de concordância (d) e de confiança (c) foram utilizados para avaliar a confiabilidade das correlações estatísticas. O índice de concordância (d), descrito pela Equação 2 (Willmott, 1981), varia de 0 a 1 e representa o quanto os valores de temperatura mínima estimada aproximam-se da temperatura mínima observada, sendo que, valores próximos a 1 indicam uma concordância perfeita.

$$
\mathrm{d}=1-\left[\frac{\sum_{\mathrm{i}=1}^{\mathrm{N}}\left(\mathrm{Y}_{\mathrm{i}}-\mathrm{X}_{\mathrm{i}}\right)^{2}}{\sum_{\mathrm{i}=1}^{\mathrm{N}}\left(\left|\mathrm{Y}_{\mathrm{i}}-\overline{\mathrm{X}}\right|+\mid \mathrm{X}_{\mathrm{i}}-\overline{\mathrm{X}}\right)^{2}}\right]
$$

Sendo, $\mathrm{Xi}=$ valores de temperatura mínima observada; $\bar{X} \bar{X}=$ é a média dos valores de temperatura mínima observada; $Y i=$ são os valores de temperaturas mínimas estimados pelo modelo; e $\mathrm{N}$ = é o número de dados de temperatura mínima.

O índice de confiança (c), proposto por Camargo e Sentelhas (1997) foi utilizado para avaliar a confiabilidade da estimativa de temperatura mínima, conforme Equação 3. O critério de interpretação dos valores de c, consta na Tabela 2.

$$
\mathrm{c}=\mathrm{r} . \mathrm{d}
$$

Tabela 2 - Critério de interpretação do índice de confiança

\begin{tabular}{c|c}
\hline Índice de confiança (c) & Desempenho \\
\hline$>0,85$ & Ótimo \\
0,76 a 0,85 & Muito bom \\
0,66 a 0,75 & Bom \\
0,61 a 0,65 & Mediano \\
0,51 a 0,60 & Sofrível \\
0,41 a 0,50 & Mau \\
$\leq 0,40$ & Péssimo \\
\hline
\end{tabular}

Foi considerado um dia com ocorrência de geada de radiação, aquele em que a temperatura mínima no abrigo foi menor ou igual a $4^{\circ} \mathrm{C}$. Este valor serviu como base para todas as estações meteorológicas, pois ao considerar as margens de erro toda a faixa de maior ocorrência da geada estaria contemplada na avaliação.

\section{Resultados e discussão}

Como resultado das análises de regressão, os maiores coeficientes de determinação $\left(R^{2}\right)$ e correlação $(r)$ estatisticamente significativos $(p<0,01)$, foram com regressão simples entre a temperatura do ar da $00 \mathrm{~h} \mathrm{TMG}$ do dia anterior (00Ti-1) com a temperatura mínima do dia presente (Tmin i), conforme Tabela 3.

Os valores dos coeficientes de determinação $\left(R^{2}\right)$ indicam que a temperatura mínima do dia presente (Tmin i), é explicada de $69 \%$ a $78 \%$ pela temperatura a $00 \mathrm{~h}$ (TMG) do dia anterior (Ti-1). As estações que apresentaram os maiores índices foram Chapecó $(0,78)$ e Campos Novos $(0,69)$, seguidos por São Joaquim $(0,67)$ e Lages $(0,66)$.

Os gráficos de dispersão em torno da reta 1:1 dos pontos entre as duas citadas variáveis meteorológicas demonstram que os resultados tendem a ser superestimados em todas as estações, conforme apresentados na Figura 1.

Vieira Júnior et al. (2009) observaram que as deficiências na modelagem de elementos climáticos se dão em razão da interação entre eles, assim como, fatores como a altitude, tem grande importância na previsão da temperatura mínima, corroborando com Bootsma (1976 apud GALVANI et al., 2000), onde afirmou que a nebulosidade juntamente com a velocidade do vento, são responsáveis por $74 \%$ da variação da temperatura mínima no abrigo e na relva.

A Tabela 4 apresenta a avaliação da estimativa da temperatura mínima (Tmin i) pelos modelos obtidos para cada local em estudo. Na associação dos resultados dos coeficientes de correlação (r) e de concordância (d), nota-se que as estimativas do modelo tiveram um desempenho 'muito bom'.

$\mathrm{Na}$ análise comparativa entre os municípios, Chapecó e Campos Novos obtiveram os melhores resultados, seguidos por São Joaquim e Lages, respectivamente. Assim, a temperatura mínima registrada a $00 \mathrm{~h}$ (TMG) respondeu por $65 \%$ a $72 \%$ da temperatura mínima do dia presente (Tmin i), com uma variabilidade de 2,4 a $2,6^{\circ} \mathrm{C}$.

Filho et al. (2006) analisaram a correlação da temperatura média do ar para as cidades do Rio Grande do Sul, através de modelos elaborados a partir da latitude, longitude e altitude. Concluíram que a latitude e longitude não apresentaram uma correlação significativa com a temperatura média do ar, ao contrário da altitude que apresentou maior influência nos resultados. Massignam e Dittrich (1998) também obtiveram resultados semelhantes ao testar um modelo baseado na altitude, latitude e longitude para previsão mensal de ocorrência de geadas em Santa Catarina.

Bardin et al. (2010) analisaram as variações de temperaturas mínimas e máximas no circuito das frutas no 
Tabela 3 - Resultado das análises de regressão linear entre a temperatura do ar da 00 h (TMG) do dia anterior (Ti1) com a temperatura mínima do dia presente (Tmin i)

\begin{tabular}{c|c|c|c|c|c}
\hline Local & $\mathrm{R}^{2}$ & $\mathrm{r}$ & valor-p & $\mathrm{F}$ & Equação \\
\hline $\begin{array}{c}\text { Campos } \\
\text { Novos }\end{array}$ & 0,69 & 0,83 & $1,2 \mathrm{E}-28$ & 3460 & $\begin{array}{c}\text { Tmin i }=-2,399+0,885 .(00 \mathrm{Ti}-1) \pm \\
2,50\end{array}$ \\
\hline Lages & 0,66 & 0,81 & $1,7 \mathrm{E}-56$ & 3057 & $\begin{array}{c}\text { Tmin i }=-3,57+0,9622 .(00 \mathrm{Ti}-1) \pm \\
2,54\end{array}$ \\
\hline Chapecó & 0,78 & 0,89 & 0 & 5280 & $\begin{array}{c}\text { Tmin i }=-2,944+0,9365 .(00 \mathrm{Ti}-1) \pm \\
2,30\end{array}$ \\
\hline São Joaquim & 0,67 & 0,86 & $1,7 \mathrm{E}-63$ & 3260 & $\begin{array}{c}\text { Tmin i }=-1,978+0,8742 . \\
2,63\end{array}$ \\
\hline
\end{tabular}

r=coeficiente de correlação; $\mathrm{R}^{2}=$ coeficiente de determinação.
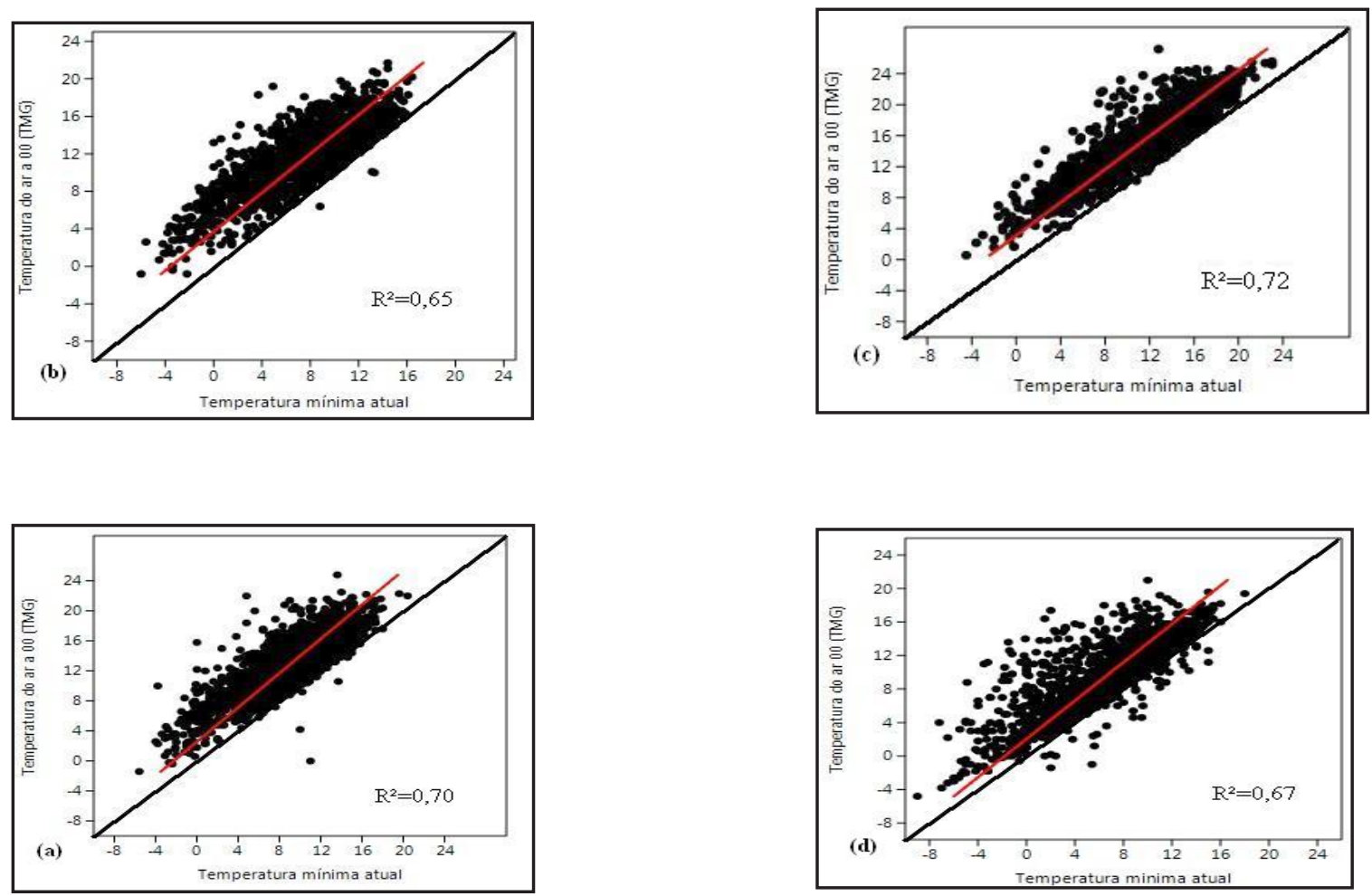

Figura 1 - Relação entre temperatura do dia anterior à $00 \mathrm{~h}$ (TMG) e temperatura mínima do dia presente (Tmin i) em Campos Novos (1a), Lages (1b), Chapecó (1c) e São Joaquim (1d).

Tabela 4 - Avaliação da estimativa da temperatura mínima utilizando dados de temperatura do ar a 00 h TMG

\begin{tabular}{c|c|c|c|c|c|c}
\hline Município & $\mathrm{R}^{2}$ & $\mathrm{r}$ & $\mathrm{d}$ & $\mathrm{EPE}$ & $\mathrm{c}$ & Desempenho \\
\hline Campos & 0,70 & 0,84 & 0,98 & 2,5 & 0,83 & Muito Bom \\
Novos & 0,65 & 0,81 & 0,98 & 2,5 & 0,78 & Muito Bom \\
Lages & 0,72 & 0,85 & 0,99 & 2,4 & 0,84 & Muito Bom \\
Chapecó & 0,67 & 0,83 & 0,98 & 2,6 & 0,81 & Muito Bom \\
São Joaquim & & & & & & \\
\hline
\end{tabular}

$r=$ coeficiente de correlação; $R^{2}=$ coeficiente de determinação; $d=$ =índice de confiança; $E P E=$ erro padrão de estimativa. 
estado de São Paulo e constataram que as variações de temperaturas máximas e mínimas, estão diretamente associadas a altitude, sendo que, quanto mais alto a localidade, maior a variação que no estudo chegou a $1,0^{\circ} \mathrm{C}$ para máximas e $0,8^{\circ} \mathrm{C}$ para mínimas a cada 100 $\mathrm{m}$. Como a diferença de altitude na região de estudo foi pequena, os autores especificaram que no inverno a entrada das massas de ar polar tornaram quase nulas estas diferenças.

Os valores observados e estimados pelos modelos somente em dias de ocorrências de geada de radiação (temperaturas mínimas $\leq 4^{\circ} \mathrm{C}$ ) no período de maio a agosto de 2014 e 2015, são apresentados na Figura 2 para cada uma das localidades analisadas no estudo.

O modelo para Chapecó obteve maior precisão nas estimativas, de forma que Chapecó obteve $88,9 \%$ de acerto, seguida por Lages 84,9\%, São Joaquim 80,9\% e Campos Novos com 64,3\%. A maioria dos dados observados para os municípios ficaram dentro dos limites de erro padrão de estimativa (EPE). Dentre todos os dias analisados, em São Joaquim houve nove situações onde a temperatura mínima estimada esteve abaixo da observada, e apenas uma para as demais localidades, ratificando a tendência dos modelos de superestimarem os valores, conforme apresentado na Figura 1. Mohanty et al. (1997) testaram modelos para a previsão de temperaturas máximas durante o verão e mínimas durante o inverno em Nova Deli, na Índia, e obtiveram 65\% de precisão com erro padrão de $\pm 1{ }^{\circ} \mathrm{C}$ e de $90 \%$ com erro padrão de $\pm 2{ }^{\circ} \mathrm{C}$.

Em regiões de relevo mais acidentado, como no caso de São Joaquim, o ar frio mais denso desloca-se pelas encostas e deposita-se nos vales, aumentando a umidade relativa e a ocorrência de inversões térmicas. Já em locais mais abertos e planos, como nas demais localidades analisadas, outros fatores tem maior relevância, como exemplo, o vento, que por advecção é responsável por remover a camada de ar mais frio que se deposita próximo ao solo e diminuindo a possibilidade do ar atingir a saturação de vapor d'água.

Galvani et al. (2000) demonstraram que a temperatura observada a 00 h (TMG) teve $82,5 \%$ de influência na temperatura mínima na cidade de Maringá-PR, enquanto a nebulosidade respondeu por 13,8\%. Significa que a nebulosidade foi responsável por um acréscimo de até 0,6 ${ }^{\circ} \mathrm{C}$ na temperatura mínima, considerando a condição de céu encoberto no momento de observação. Quando os pesquisadores repetiram o experimento utilizando apenas a temperatura do ar a $00 \mathrm{~h}$ (TMG), o valor subiu para $83 \%$ de influência desta, na temperatura mínima do dia subsequente. Assim, defenderam a adoção do modelo baseado na temperatura do ar medida a $00 \mathrm{~h}$ (TMG) por ser de fácil observação e necessitar de apenas uma variável, além de que a nebulosidade é um fator subje-

Figura 2 - Comparação da temperatura mínima estimada com a observada em dias com ocorrência de geada (Tmin $\leq 4^{\circ} \mathrm{C}$ ) para os municípios de Campos Novos (2a), Chapecó (2b), Lages (2c) e São Joaquim (2 d) no período de maio a agosto de 2014 e 2015.
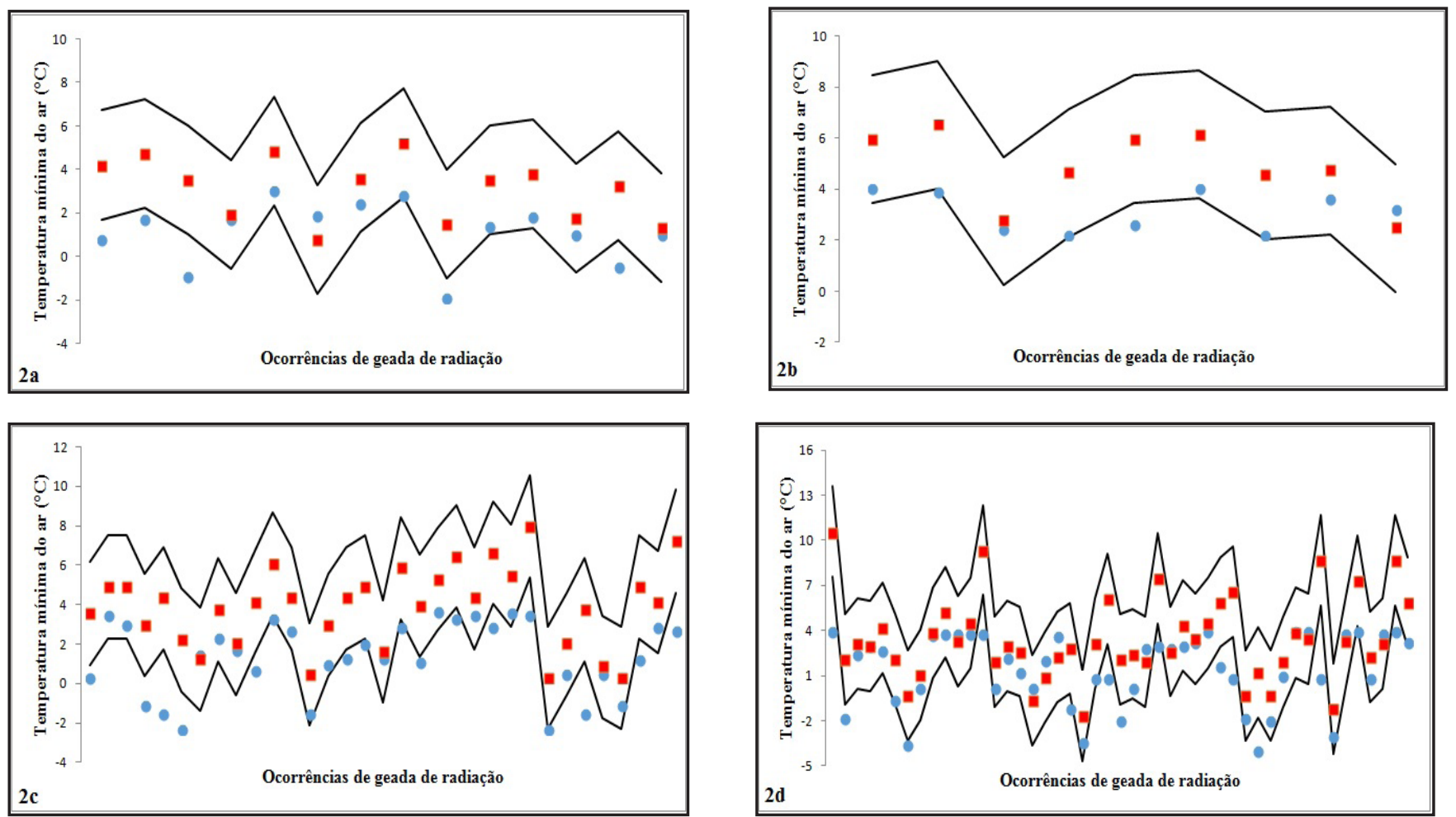

- Observada 
Tabela 5 - Estimativa da temperatura mínima do dia subsequente, baseado na temperatura do ar da 00 h (TMG)

\begin{tabular}{|c|c|c|c|c|c|c|c|c|}
\hline \multirow{4}{*}{$\begin{array}{l}\text { Temperatura } \\
00 \mathrm{~h} \mathrm{TMG}\left({ }^{\circ} \mathrm{C}\right)\end{array}$} & \multicolumn{8}{|c|}{ Temperatura minima do dia subsequente $\left({ }^{\circ} \mathrm{C}\right)$} \\
\hline & \multirow{2}{*}{\multicolumn{2}{|c|}{$\begin{array}{c}\text { São Joaquim } \\
\text { Limites }\end{array}$}} & \multirow{2}{*}{\multicolumn{2}{|c|}{$\begin{array}{c}\text { Lages } \\
\text { Limites } \\
\end{array}$}} & \multirow{2}{*}{\multicolumn{2}{|c|}{$\begin{array}{l}\text { Chapecó } \\
\text { Limites }\end{array}$}} & \multirow{2}{*}{\multicolumn{2}{|c|}{$\begin{array}{c}\text { Campos novos } \\
\text { Limites }\end{array}$}} \\
\hline & & & & & & & & \\
\hline & - & + & - & + & - & + & - & + \\
\hline$-8,5$ & $-11,6$ & $-6,7$ & $-14,2$ & $-9,2$ & $-13,2$ & $-8,6$ & $-11,9$ & $-7,9$ \\
\hline-8 & $-11,2$ & $-6,3$ & $-13,8$ & $-8,8$ & $-12,7$ & $-8,1$ & $-11,5$ & $-7,5$ \\
\hline$-7,5$ & $-10,8$ & $-5,9$ & $-13,3$ & $-8,3$ & $-12,3$ & $-7,7$ & $-11,0$ & $-7,0$ \\
\hline-7 & $-10,3$ & $-5,4$ & $-12,8$ & $-7,8$ & $-11,8$ & $-7,2$ & $-10,6$ & $-6,6$ \\
\hline$-6,5$ & $-9,9$ & $-5,0$ & $-12,3$ & $-7,3$ & $-11,3$ & $-6,7$ & $-10,2$ & $-6,2$ \\
\hline-6 & $-9,5$ & $-4,6$ & $-11,8$ & $-6,8$ & $-10,9$ & $-6,3$ & $-9,7$ & $-5,7$ \\
\hline$-5,5$ & $-9,0$ & $-4,1$ & $-11,4$ & $-6,4$ & $-10,4$ & $-5,8$ & $-9,3$ & $-5,3$ \\
\hline-5 & $-8,6$ & $-3,7$ & $-10,9$ & $-5,9$ & $-9,9$ & $-5,3$ & $-8,8$ & $-4,8$ \\
\hline$-4,5$ & $-8,2$ & $-3,3$ & $-10,4$ & $-5,4$ & $-9,5$ & $-4,9$ & $-8,4$ & $-4,4$ \\
\hline-4 & $-7,8$ & $-2,9$ & $-9,9$ & $-4,9$ & $-9,0$ & $-4,4$ & $-7,9$ & $-3,9$ \\
\hline$-3,5$ & $-7,3$ & $-2,4$ & $-9,4$ & $-4,4$ & $-8,5$ & $-3,9$ & $-7,5$ & $-3,5$ \\
\hline 3 & $-1,7$ & 3,2 & $-3,2$ & 1,8 & $-2,4$ & 2,2 & $-1,7$ & 2,3 \\
\hline$-2,5$ & $-6,5$ & $-1,6$ & $-8,5$ & $-3,5$ & $-7,6$ & $-3,0$ & $-6,6$ & $-2,6$ \\
\hline-2 & $-6,0$ & $-1,1$ & $-8,0$ & $-3,0$ & $-7,1$ & $-2,5$ & $-6,2$ & $-2,2$ \\
\hline$-1,5$ & $-5,6$ & $-0,7$ & $-7,5$ & $-2,5$ & $-6,6$ & $-2,0$ & $-5,7$ & $-1,7$ \\
\hline-1 & $-5,2$ & $-0,3$ & $-7,0$ & $-2,0$ & $-6,2$ & $-1,6$ & $-5,3$ & $-1,3$ \\
\hline 0 & $-4,3$ & 0,6 & $-6,1$ & $-1,1$ & $-5,2$ & $-0,6$ & $-4,4$ & $-0,4$ \\
\hline 1 & $-3,4$ & 1,5 & $-5,1$ & $-0,1$ & $-4,3$ & 0,3 & $-3,5$ & 0,5 \\
\hline 1,5 & $-3,0$ & 1,9 & $-4,6$ & 0,4 & $-3,8$ & 0,8 & $-3,1$ & 0,9 \\
\hline 2 & $-2,6$ & 2,3 & $-4,1$ & 0,9 & $-3,4$ & 1,2 & $-2,6$ & 1,4 \\
\hline 2,5 & $-2,1$ & 2,8 & $-3,7$ & 1,3 & $-2,9$ & 1,7 & $-2,2$ & 1,8 \\
\hline 3 & $-1,7$ & 3,2 & $-3,2$ & 1,8 & $-2,4$ & 2,2 & $-1,7$ & 2,3 \\
\hline 3,5 & $-1,3$ & 3,6 & $-2,7$ & 2,3 & $-2,0$ & 2,6 & $-1,3$ & 2,7 \\
\hline 4 & $-0,8$ & 4,1 & $-2,2$ & 2,8 & $-1,5$ & 3,1 & $-0,9$ & 3,1 \\
\hline 4,5 & $-0,4$ & 4,5 & $-1,7$ & 3,3 & $-1,0$ & 3,6 & $-0,4$ & 3,6 \\
\hline 5 & 0,0 & 4,9 & $-1,3$ & 3,7 & $-0,6$ & 4,0 & 0,0 & 4,0 \\
\hline 5,5 & 0,5 & 5,4 & $-0,8$ & 4,2 & $-0,1$ & 4,5 & 0,5 & 4,5 \\
\hline 6 & 0,9 & 5,8 & $-0,3$ & 4,7 & 0,4 & 5,0 & 0,9 & 4,9 \\
\hline 6,5 & 1,3 & 6,2 & 0,2 & 5,2 & 0,8 & 5,4 & 1,4 & 5,4 \\
\hline 7 & 1,7 & 6,6 & 0,7 & 5,7 & 1,3 & 5,9 & 1,8 & 5,8 \\
\hline 7,5 & 2,2 & 7,1 & 1,1 & 6,1 & 1,8 & 6,4 & 2,2 & 6,2 \\
\hline 8 & 2,6 & 7,5 & 1,6 & 6,6 & 2,2 & 6,8 & 2,7 & 6,7 \\
\hline 8,5 & 3,0 & 7,9 & 2,1 & 7,1 & 2,7 & 7,3 & 3,1 & 7,1 \\
\hline 9 & 3,5 & 8,4 & 2,6 & 7,6 & 3,2 & 7,8 & 3,6 & 7,6 \\
\hline 9,5 & 3,9 & 8,8 & 3,1 & 8,1 & 3,7 & 8,3 & 4,0 & 8,0 \\
\hline 10 & 4,3 & 9,2 & 3,6 & 8,6 & 4,1 & 8,7 & 4,5 & 8,5 \\
\hline 10,5 & 4,8 & 9,7 & 4,0 & 9,0 & 4,6 & 9,2 & 4,9 & 8,9 \\
\hline 11 & 5,2 & 10,1 & 4,5 & 9,5 & 5,1 & 9.7 & 5,3 & 9,3 \\
\hline 11,5 & 5,6 & 10,5 & 5,0 & 10,0 & 5,5 & 10,1 & 5,8 & 9,8 \\
\hline 12 & 6,1 & 11,0 & 5,5 & 10,5 & 6,0 & 10,6 & 6,2 & 10,2 \\
\hline 12,5 & 6,5 & 11,4 & 6,0 & 11,0 & 6,5 & 11,1 & 6,7 & 10,7 \\
\hline 13 & 6,9 & 11,8 & 6,4 & 11,4 & 6,9 & 11,5 & 7,1 & 11,1 \\
\hline 13,5 & 7,4 & 12,3 & 6,9 & 11,9 & 7,4 & 12,0 & 7,5 & 11,5 \\
\hline 14 & 7,8 & 12,7 & 7,4 & 12,4 & 7,9 & 12,5 & 8,0 & 12,0 \\
\hline 14,5 & 8,2 & 13,1 & 7,9 & 12,9 & 8,3 & 12,9 & 8,4 & 12,4 \\
\hline 15 & 8,7 & 13,6 & 8,4 & 13,4 & 8,8 & 13,4 & 8,9 & 12,9 \\
\hline 15,5 & 9,1 & 14,0 & 8,8 & 13,8 & 9,3 & 13,9 & 9,3 & 13,3 \\
\hline 16 & 9,5 & 14,4 & 9,3 & 14,3 & 9,7 & 14,3 & 9,8 & 13,8 \\
\hline
\end{tabular}


tivo e dependente da interpretação de cada observador.

Em Santa Catarina, as noites de inverno são caracterizadas pela baixa nebulosidade em virtude da maior frequência da atuação de massas de ar frio e/ou polar, o que diminui a influência desta na temperatura mínima do dia subsequente (Araújo et al., 2009), já que, a nebulosidade diminui a perda de calor por radiação de ondas longas evitando decréscimos muito acentuados da temperatura do ar. Como em São Joaquim a temperatura é climaticamente menor por estar a uma altitude bem superior em relação às demais localidades e associado com a disponibilidade de vapor d'água da região, o ar atinge com maior frequência o nível de condensação (nível em que é atingido o ponto de orvalho). Assim, esta condição favorece uma frequência relativamente maior de nebulosidade e, principalmente nevoeiros.

Silva e Sentelhas (2001) pesquisaram a relação entre a temperatura do abrigo e a medida na relva em noites de geada para Santa Catarina, concluindo que a diferença média entre estes dois ambientes, variou de 2,1 a $4,8{ }^{\circ} \mathrm{C}$, de acordo com as condições atmosféricas associadas à topografia e as características da superfície. Como as estações meteorológicas são padronizadas, a nebulosidade, a umidade do ar e a velocidade do vento foram os principais responsáveis pela variação no balanço energético de radiação, o que explica a diferença entre a temperatura no abrigo e na relva. Ao considerarem a relação desta variação com o risco de geada, notaram que para algumas cidades a faixa com probabilidade de ocorrência é maior do que em relação às outras. Em São Joaquim, o risco de geada ultrapassou a variação de até $10^{\circ} \mathrm{C}$, diferente das demais cidades onde o risco não ultrapassa $7^{\circ} \mathrm{C}$.

O cruzamento da faixa de maior ocorrência para cada cidade com o seu respectivo modelo de estimativa baseado na temperatura mínima do ar a 00 h (TMG), permitiu elaborar uma tabela para estimar a temperatura mínima do dia subsequente e, assim, a possibilidade de ocorrência de geada de radiação Tabela 5. De posse destas informações de fácil obtenção, podem-se tomar medidas em curto prazo, necessárias para evitar/minimizar os danos decorrentes da geada de radiação.

Embora uma situação sinótica favorável para a ocorrência de geada possa ser prevista com relativa facilidade e antecedência razoável, a formação de geada em uma região com distintos micros ou mesoclimas requerem um maior conhecimento e ferramentas mais aprimoradas (SELUCHI, 2009). Neste contexto, as características orográficas e topográficas podem tornar a previsão baseada em modelagem numérica de mesoescala mais trabalhosa, pela dificuldade em incluí-las nos modelos. Assim, métodos estatísticos podem ser requeridos para previsão em curto prazo de elementos e fenômenos meteorológicos em locais específicos.

\section{Conclusões}

A temperatura do ar a $00 \mathrm{~h}$ (TMG) é a melhor variável preditora para a temperatura mínima do dia seguinte (Tmin i).

Em eventos de geada branca $\left(\operatorname{Tmin} \leq 4^{\circ} \mathrm{C}\right)$ o acerto esteve entre $64,3 \%$ e $88,9 \%$.

Os modelos estatísticos para previsão de temperatura mínima apresentaram um desempenho 'muito bom', permitindo que a geada de radiação possa ser prevista da maneira prática e confiável.

\section{Referências}

AGUIAR, D.; MENDONÇA, M. Climatologia das geadas em Santa Catarina. In: SIMPÓSIO BRASILEIRO DE DESASTRES NATURAIS, 1., 2004, Florianópolis. Anais... GEDN/UFSC, 2004. P.762-773.

ARAÚJOS, C. E. S de; et al. Previsão de geadas em Santa Catarina. Florianópolis - SC: Epagri, 2009. 11p. (Relatório do Projeto PREVISÃO DE EVENTOS EXTREMOS NO SUL DO BRASIL - FINEP 14). Disponível em < http:// www.ciram.com.br/finep14/recursos/Rel_Finep14_ Geada.pdf>

BARDIN, L.; JUNIOR, M. J. P.; MORAES, J. F. L. Estimativa das temperaturas máximas e mínimas do ar para a região do Circuito das Frutas, SP. Revista Brasileira de Engenharia Agrícola e Ambiental, Campina Grande, v.14, n.6, p. 618-624, 2010.

BRIXNER, G. F.; et. Risco de geada e duração dos subperíodos fenológicos da 'Cabernet Sauvignon' na região da Campanha. Revista Brasileira de Engenharia Agrícola e Ambiental, Campina Grande v.18, n.2, p. 217-224, 2014.

CAMARGO, A. P.; SENTELHAS, P. C. Avaliação do desempenho de diferentes métodos de estimativa da evapotranspiração potencial no Estado de São Paulo. Revista Brasileira de Agrometeorologia, Santa Maria, v. 5, p. 89-97, 1997.

FILHO, A. C.; et al. Altitude e coordenadas geográficas na estimativa da temperatura mínima média decendial do ar no Estado do Rio Grande do Sul. Pesquisa Agropecuária Brasileira, Brasília, v.41, n6, p.893-901, 2006.

GALVANI, E.; et al. Modelo de estimativa de temperatura mínima do ar para a região de Maringá-PR. Revista Brasileira de Agrometeorologia, Santa Maria, v.8, n.1, p. 105-110, 2000. 
MASSIGNAM, A. M.; DITTRICH, R. C.; Estimativa do número e da probabilidade mensal de ocorrência de geadas para o estado de Santa Catarina. Revista Brasileira de Agrometeorologia, Santa Maria, v.6, n.2, p.213-220, 1998.

MOHANTY, U. C.; et al. Forecasting minimum temperature during winter and maximum temperature during Summer at Delhi. Meteorology Application, v.4, p.37-48, 1997.

PERISSATO, S. M.; et al. Efeito das geadas em culturas energéticas. Revista Brasileira de Energias Renováveis, v.1, p.49-58, 2013.

RAMOS, C. M.; et al. Modelagem da variação horária da temperatura do ar em Petrolina-PE e Botucatu-SP. Revista Brasileira de Engenharia Agrícola e Ambiental, Campina Grande v.15, n.9, p. 959-965, 2011.

SELUCHI, M. E. Geadas e friagens. In: CAVALCANTI, I. F. A. et al. (Org.). Tempo e clima no Brasil. São Paulo: Oficina de Textos, 2009. p.149-168.

SENTELHAS, P. C.; ANGELOCCI, L. R. Entendendo a geada, 2012. Disponível em <http://www.climaonline. com.br/artigo03. php.> Acesso em: 14 março 2016.

SILVA, J. G.; SENTELHAS, P. C. Diferença de temperatura mínima do ar medida no abrigo e na relva e probabilidade de sua ocorrência em eventos de geada no Estado de Santa Catarina. Revista Brasileira de Agrometeorologia, Santa Maria, v.9, n.1, p. 9-15, 2001.

VIEIRA JÚNIOR, P. A.; et al. Previsões meteorológicas do Modelo Eta para subsidiar o uso de modelos de previsões agrícolas no Centro-Sul do Brasil. Ciência Rural, Santa Maria, v.39, n.2, p. 412-420, 2009.

WILLMOTT, C. J. On the validation of models. Physical Geography, Oxford, v.2, n.2, p.184-194, 1981.

\section{APÊNDICE}

Tabela 1 - Estimativa da temperatura mínima do dia subsequente, baseado na temperatura do ar da $00 \mathrm{~h}$ (TMG). 